\title{
Peanut Oral Immunotherapy: a Current Perspective
}

\author{
Meera Patrawala ${ }^{1,2} \cdot$ Jennifer Shih ${ }^{1,2} \cdot$ Gerald Lee $^{1,2} \cdot$ Brian Vickery $^{2}$ \\ Published online: 20 April 2020 \\ (C) Springer Science+Business Media, LLC, part of Springer Nature 2020
}

\begin{abstract}
Purpose of the Review Peanut oral immunotherapy (OIT) is one of the most studied experimental therapies for food allergy. With the recently FDA-approved peanut product, Palforzia, the goal of this article is to review the most recent data from clinical trials, discuss recent trends, and anticipate future developments.

Recent Findings The latest research suggests that peanut OIT could be a promising option for peanut-allergic patients, with the majority of participants in research studies achieving the primary efficacy endpoint of desensitization, as well as sustained unresponsiveness in select populations. Some studies also showed improvements in food allergy-related quality of life. However, peanut OIT is not without risk or side effects, including potentially serious allergic reactions.

Summary Future research will need to evaluate the short- and long-term effectiveness of the therapy in the real-world setting, predictors of important treatment outcomes, and the use of adjunctive therapies that may mitigate some of these allergic reactions.
\end{abstract}

Keywords Peanut allergy $\cdot$ Oral immunotherapy $\cdot$ Desensitization $\cdot$ Sustained unresponsiveness $\cdot$ Food allergy $\cdot$ Oral food challenge

\section{Introduction}

Food allergy affects about $5 \%$ of adults and $8 \%$ of children, with peanut allergy affecting about $2 \%$ of the population [1]. The main treatment approach recommended by U.S. guidelines has been strict allergen avoidance and early treatment with rescue medications if reactions occur $[2,3]$. However, peanut allergy has a negative impact on quality of life and healthcare costs. These factors highlight an urgent need for

This article is part of the Topical Collection on Food Allergy

Meera Patrawala

meera.mehta@emory.edu

Jennifer Shih

Jennifer.a.shih@emory.edu

Gerald Lee

gerald.b.lee@emory.edu

Brian Vickery

brian.p.vickery@emory.edu

1 Emory University School of Medicine, 1400 Tullie Road NE 5th floor, Atlanta, GA 30329, USA

2 Children's Healthcare of Atlanta, 1400 Tullie Road NE 5th floor, Atlanta, GA 30329, USA alternative options for this potentially life-threatening condition. Currently, there are ongoing clinical research trials to evaluate alternative therapies for peanut allergy. Peanut oral immunotherapy (OIT) is one of the most investigated alternative options with a recent United States Food and Drug Administration (FDA) approval in early 2020 for a peanut OIT product, Palforzia. In this review, we examine data from peanut OIT clinical trials and discuss the recent trends and future developments in peanut OIT.

\section{Peanut Oral Immunotherapy}

\section{General Protocol}

Peanut OIT is currently considered an investigational therapy for peanut-allergic patients. The overall goal of OIT is to facilitate a state of desensitization by exposing the participant to increasing doses of peanut protein. While considerable differences exist between clinical trial protocols, the desensitization process is conceptually similar. Participants are initially selected based on specific criteria such as a physician-confirmed history of peanut allergy (though this is not universally the case), along with test results that support that history. Patients have typically been excluded if they had a history of 
a life-threatening reaction, poorly controlled asthma, suspicion of eosinophilic gastrointestinal disease, or other factors that would hinder their ability to cooperate with the study [4]. Importantly, high-specific IgE levels do not disqualify participants from these trials. Some but not all studies then uses double-blind, placebo-controlled food challenges (DBPCFC) to confirm eligibility. On a subsequent day, qualifying participants then consume an initial starting dose below the participant's threshold, with rapid same-day dose escalation until the participant has a reaction or reaches the maximum dose for that day, typically in the range of 5-50 $\mathrm{mg}$ of protein. The participant will return at scheduled intervals for the build-up phase with the goal of increasing the daily dose. Ultimately, the participant reaches a maintenance dose (or highest dose tolerated) which will be continued daily for a specified period [4].

\section{Efficacy vs Effectiveness}

While there is no single definition of efficacy, in clinical trials this may be defined as achieving specific endpoints. The primary efficacy endpoint of most peanut OIT is desensitization, which can be defined as a transient shift upwards in the reactive allergen dose threshold requiring continued consumption of the allergen. It may be a state of clinical remission (also known as sustained unresponsiveness (SU)), based on a successful challenge after cessation of therapy [5]. Based on these definitions, experimental studies have demonstrated the efficacy of OIT in the treatment of peanut allergy [6-11].

On the other hand, effectiveness describes how the therapy performs in real-world settings outside of the clinical trial environment. Whereas efficacy is usually measured in a trial by an exit DBPCFC, the effectiveness of desensitization could be observed with a reduction in the frequency or severity of accidental reactions. There is some sparse data available from real-world settings, but in general, the lack of real-world effectiveness data in peanut OIT was cited in a recent review by the Institute for Clinical and Economic Review [12]. Wasserman et al. published a retrospective review of 270 patients (ages 4-18 years) who received peanut OIT [7••]. 96.7\% of patients had a history consistent with an IgE-mediated reaction, with testing that supported a peanut allergy; most patients did not have confirmatory food challenges prior to commencing OIT. The protocol included a target dose of $3000 \mathrm{mg}$ of peanut protein followed by a $6000 \mathrm{mg}$ peanut protein oral food challenge (OFC) and subsequent maintenance dosing of $2000 \mathrm{mg}$ for 3 years [7••].

Seventy-nine percent completed the escalation phase to maintenance, $18 \%$ discontinued therapy before reaching maintenance, and $12 \%$ dropped out during the maintenance phase. The most common reason for stopping escalation was due to eosinophilic esophagitis (EOE) like oral immunotherapy-related syndrome and epinephrine-treated reactions [7••]. Adverse events (AEs) requiring epinephrine occurred 100 times in 63/270 (23\%) of patients. Peanut IgE decreased by $65 \%$ over 3 years of maintenance with $6.5 \%$ achieving SU, which was defined as tolerating $6000 \mathrm{mg}$ of PP (24 peanuts) after avoiding for 30 days [7 • $]$. These realworld data provide a glimpse at how peanut OIT may work outside of clinical trial protocols, however, there were some limitations. These include a variety of peanut products used, no formal monitoring of the frequency and severity of accidental reactions, an appreciable dropout rate of $18 \%$ prior to reaching maintenance, and lack of extended follow-up. With the recent FDA approval of a scalable standardized peanut OIT product, observational data from patients in routine clinical practice will need to be carefully analyzed to determine real-world effectiveness (e.g., reduced frequency/severity following accidental peanut exposure) in both the short and long term.

Theoretically, desensitization has the potential to address the needs of food allergic patients and caregivers by increasing the threshold of peanut protein required to trigger an allergic reaction. Therefore, while continuing a strict peanut-free diet on treatment, patients and caregivers may feel as though they are freer to participate in normal daily activities with less anxiety about peanut exposure once desensitized.

\section{Risks and Benefits of Peanut OIT}

While peanut OIT clinical trials have shown data to suggest efficacy, as with all therapies, the risks and benefits of treatment are important to review [6-11].

Among the adverse events (AEs) that can occur, the most concerning is anaphylaxis, which in its worst form has the potential to be life-threatening. Chu et al. recently published a meta-analysis that included 12 randomized, controlled peanut OIT trials with 1041 participants comparing peanut OIT versus no peanut OIT [13]. It showed high-certainty evidence that peanut OIT increased the risk of anaphylaxis (RR 3.12; 95\% CI 1.76-5.55), the frequency of anaphylaxis (IRR 2.72; 95\% CI 1.57-4.72), and the use of epinephrine during buildup and maintenance phases (RR 2.21; 95\% CI 1.27-3.83). Additionally, it showed moderate-certainty evidence that peanut OIT increased serious AEs (RR 1.92; 95\% CI 1.00-3.66), although, in this analysis, all cases of anaphylaxis were considered to be serious, independent of whether they were considered as such by the original study team. The authors estimated that peanut oral immunotherapy was associated with 151 more episodes of anaphylaxis per 1000 participants than would be expected in patients practicing avoidance [13]. Therefore, it is important to be cognizant that the therapy itself can cause AEs, including allergic reactions. A key future research goal is to determine whether there are biological or demographic characteristics among those experiencing anaphylaxis and serious adverse events that could identify the 
at-risk population and ideally predict these events and/or identify such patients as candidates for other approaches (e.g., cotreatment with a biologic, very slow updosing, continued avoidance). Another important goal of future research is a better understanding of the augmentation factors like exercise, viral infection, menses, and others that have been repeatedly shown to play a role in many such events $[14,15]$.

Regarding the benefits, clinical trials have demonstrated desensitization, sustained unresponsiveness, and an improvement in quality of life (QoL). We will discuss this in further detail.

\section{Peanut OIT Clinical Trials Summary}

Table 1 summarizes the results of relevant peanut OIT clinical trials published within the last 5 years.

The PALISADE group of clinical investigators conducted an international phase 3 randomized placebo-controlled clinical trial evaluating AR101 (a peanut-derived OIT drug) [11]. This study remains the largest peanut allergy immunotherapy trial conducted. In addition, it included patients with a history of severe or life-threatening reactions and did not use medical prophylaxis. Participants ages 4-55 years with peanut allergy who reacted during a DBPCFC conducted at screening at a challenge dose of $100 \mathrm{mg}$ or less of peanut protein were randomly assigned in a 3:1 ratio to receive active treatment with AR101 or placebo in an escalating dose program. They then received $300 \mathrm{mg}$ per day maintenance treatment for 24 weeks. In agreement with the FDA, the pre-specified primary endpoint was the proportion of those aged 4-17 years who could tolerate a challenge dose of $600 \mathrm{mg}$ or more of peanut protein on an exit DBPCFC. Of the 496 participants ages 4-17 years, $67.2 \%$ in the active treatment group were able to tolerate a $600 \mathrm{mg}$ dose compared to $4 \%$ in the placebo group in the intent to treat analysis. Severe adverse reactions (SAEs) occurred in less than $6 \%$ of the participants in the active drug group and in less than $2 \%$ of those in the placebo. Overall, systemic allergic reactions (SARs) occurred in 53 (14.2\%) of the active group and $4(3.2 \%)$ of the placebo group. Due to AEs during the intervention period, $43(11.6 \%)$ in active group and $3(2.4 \%)$ in placebo group withdrew from the study. Excluding the food challenges, epinephrine was administered in $52(14 \%)$ of participants in the active-drug group and 8 $(6.5 \%)$ in the placebo. A total of 16 participants of the active-drug group $(4.3 \%)$ withdrew from the trial due to chronic or recurrent dose-limiting gastrointestinal symptoms. Three underwent EGD and one participant was diagnosed with EOE during the trial. It is important to note that not all participants with dose limiting gastrointestinal symptoms underwent an endoscopy meaning EOE may have been underestimated. Efficacy was not demonstrated in participants older than 18 years old, likely due to the small sample size and high withdrawal rate [11].
In comparison, a more recent study by Blumchen et al. evaluated 62 participants ages 3-17 years in a double-blind, randomized, placebo-controlled (DBRPC) trial assessing the efficacy of relatively low-dose OIT [8••]. This trial evaluated whether a lower and slower dosing regimen could achieve similar outcomes to prior studies and improve safety. The dose-escalation occurred over a maximum of 14 months (median of 13 months) followed by 16 months of maintenance. This is compared to the PALISADE group in which the patient was dose escalated from 3 to $300 \mathrm{mg}$ every 2 weeks followed by a 24-week maintenance with a total duration of the study of 12 months [11]. The active group received between 125 and $250 \mathrm{mg} /$ day of peanut protein and the control group received placebo for 16 months. The primary endpoint was to evaluate the proportion of participants who could tolerate at least $300 \mathrm{mg}$ of peanut protein in the final challenge. The authors showed that $74.2 \%$ of the OIT group tolerated at least $300 \mathrm{mg}$ of peanut protein vs $16.1 \%$ in the placebo group. Treatmentrelated AEs, which were all mild-moderate in severity, occurred in $90 \%$ of the active group vs $77 \%$ of the placebo group. There was no epinephrine use for AEs. They found no difference between the groups in dropouts due to AEs, occurrence of SAEs and objective OIT-related AEs, severity of symptoms, treatment of symptoms, or worsening of preexisting atopic diseases. The dropout rate was $6.7 \%$ and no patients had development of EOE [8••]. These results suggest that a slower up-dosing schedule and lower maintenance doses than was tested in PALISADE could potentially still yield clinically meaningful desensitization with improvement in the safety profile of OIT, which if validated could help to optimize OIT regimens.

Another study similarly explored ways to optimize peanut OIT by focusing on a younger patient population. Vickery et al. evaluated 37 newly diagnosed preschool children ages 9-36 months with suspected or known peanut allergy confirmed by an entry food challenge. They were randomized to receive early-OIT (E-OIT) at goal maintenance doses of 300 or $3000 \mathrm{mg} /$ day with the primary endpoint evaluating sustained unresponsiveness at 4 weeks (4-SU) after stopping treatment. The results showed that $78 \%$ of participants achieved 4-SU with a decline in peanut-specific IgE recognizing the natural history of peanut allergy in which $\sim 20 \%$ of peanut allergy resolves in children by age 4 years [16 ${ }^{\circ}$. There were no SAEs and $95 \%$ of participants had AEs likely related to OIT more frequently during the build-up phase. Of these AEs, $85 \%$ were mild and $15 \%$ moderate. About $47 \%$ of participants required treatment with antihistamines and no one required epinephrine during dose escalation, with one requiring at home after a dose [6]. A cross-Canadian group of clinicians recently published a similarly reassuring adverse event profile in 270 young children ages 9-71 months treated with peanut OIT in their clinics, as is common practice there. Over 16 to 22 weeks, participants underwent escalation dosing with 


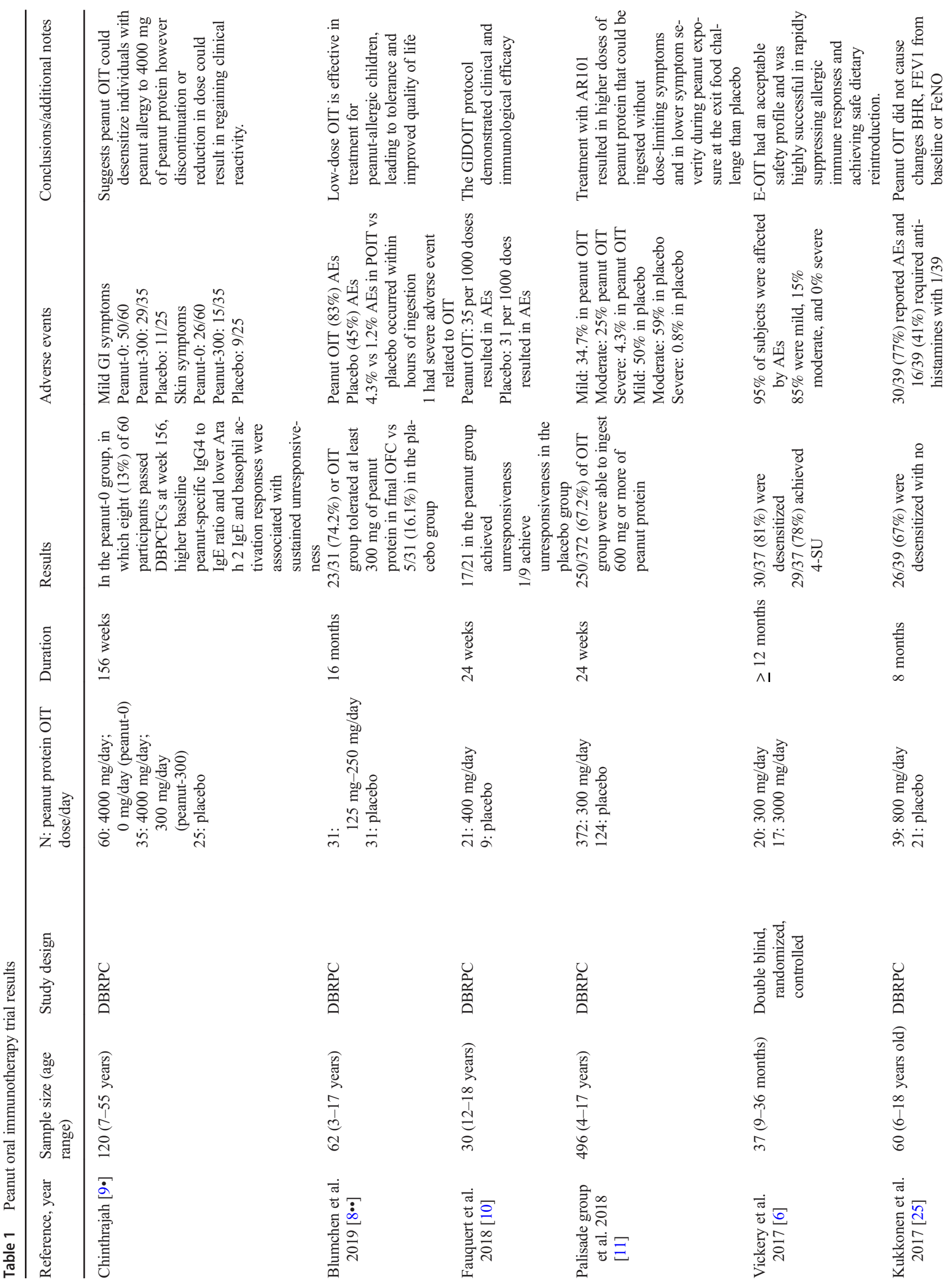




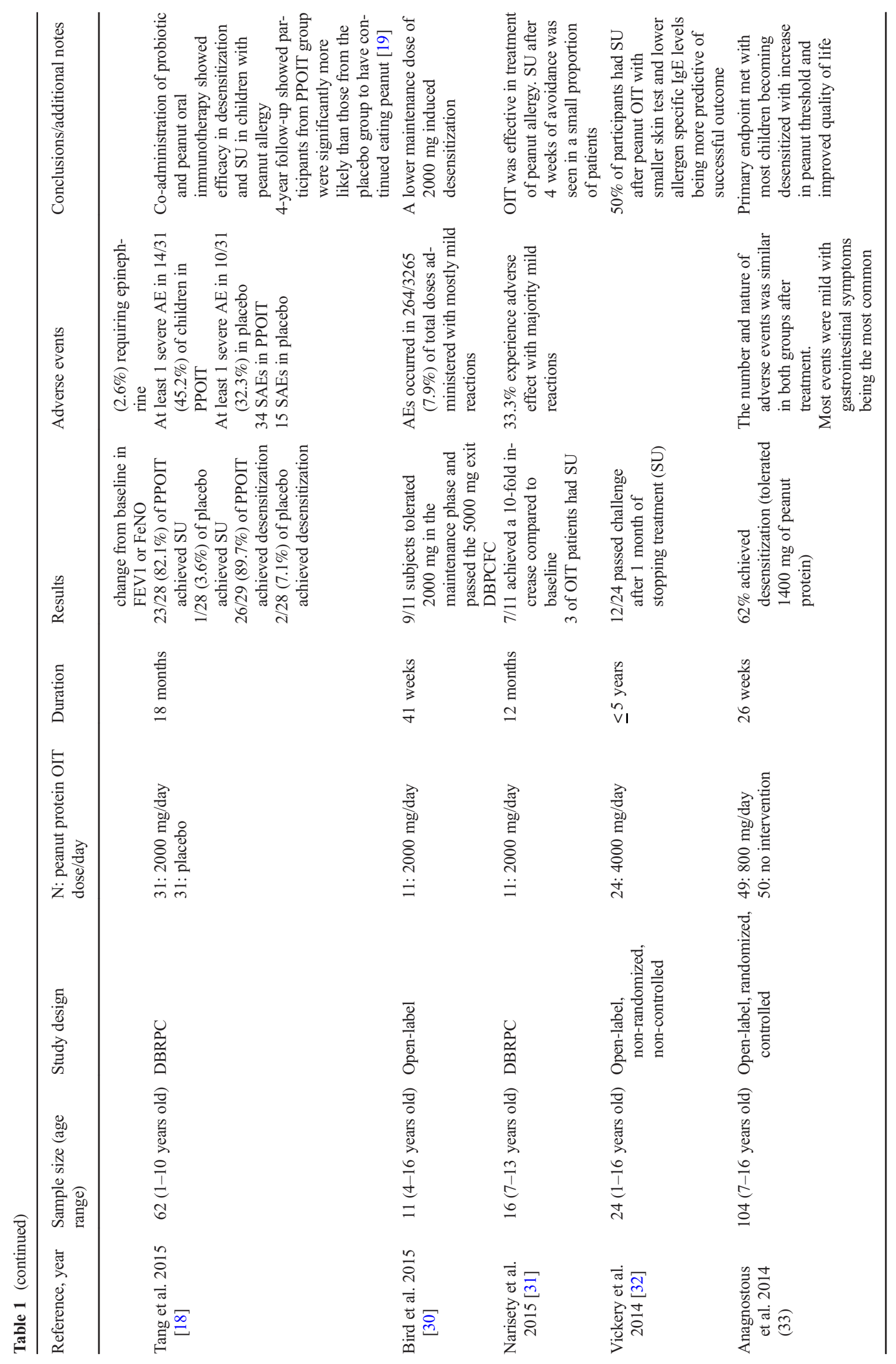


goal maintenance dose of $300 \mathrm{mg}$ of peanut protein. Of those who reached maintenance, $243,10 \%$ dropped out, $67.8 \%$ experienced reactions during build-up: $36.3 \%$ grade $1,31.1 \%$ grade 2 , and $0.4 \%$ grade $3.11 / 243(4.1 \%)$ required epinephrine. This represented $2.23 \%$ of reactions (12 of 538) and $0.029 \%$ of doses $(12$ of 41,020$)$ [17]. This data suggests that the peanut OIT is generally tolerated with few and generally, mild reactions however that life-threatening reactions, although rare, can occur [17].

In an alternative perspective, a few studies have looked to evaluate other approaches to peanut OIT in order to induce desensitization. In 2018, Fauquert et al. performed a DBRPC trial to evaluate a new modality of peanut OIT to induce desensitization in 30 peanut-allergic adolescents. Participants in the active group ingested sealed capsules of peanut bypassing the upper digestive tract for a method the authors termed gastrointestinal delivery oral immunotherapy (GIDOIT). Peanut or placebo capsules were ingested daily over 24 weeks (buildup phase) at 2-week increments from 2 to $400 \mathrm{mg}$ of peanut protein with the goal of tolerance of $400 \mathrm{mg}$. Of the participants recruited, $81 \%$ in the peanut group achieved unresponsiveness to $400 \mathrm{mg}$ of peanut protein compared to $10 \%$ in the placebo group [10]. The number of participants experiencing AEs was not different between the two groups; however, the number of AEs per patient was higher in the OIT group than the control group. The number of doses that resulted in AEs was 35 per 1000 in the intervention group vs 31 per 1000 in the placebo group. In the OIT group, 91 per 1000 doses required medication and 36 per 1000 in the placebo group. Five SAEs occurred in 4 participants in the OIT group and 0 in the placebo group [10]. Tang et al. conducted a DBRPC trial combining the probiotic Lactobacillus rhamnosus combined with peanut OIT for 18 months in 62 children ages 1-10 years with peanut allergy. The peanut OIT protocol was a 1-day rush induction phase with a build-up phase with up dosing every 2 weeks to a maintenance dose of $2 \mathrm{~g}$ of peanut protein for a total of 18 months. The active group received the probiotic with the peanut OIT (PPOIT) daily and the placebo group received placebo with placebo daily. An oral peanut DBPCFC was performed on the last day of study treatment to assess for desensitization to $4 \mathrm{~g}$ of peanut protein. Those who passed underwent another challenge at 2 weeks or more of the study treatment to assess for SU. The primary outcome assessed was SU. $82.1 \%$ of the participants treated with PPOIT and $3.6 \%$ of placebo-treated participants achieved SU. Desensitization was achieved in $89.7 \%$ of participants in the PPOIT group and $7.1 \%$ in the placebo group. At least $1 \mathrm{SAE}$ was reported in $45.2 \%$ of children in the PPOIT group compared with placebo group, however, 1 child in the PPOIT group had 13 SAEs. The number of SAEs per participant during the build-up was similar between groups; however, reactions during maintenance were more common in the PPOIT group than placebo group [18]. Because this study did not control for the effect of the probiotic, it is not possible to determine whether there was an adjunctive effect with combination therapy. In a follow-up study 4 years after treatment was stopped, participants from the PPOIT group were more likely than those from the placebo group to have continued eating peanut (67\% vs $4 \%$ ). Fifty-eight percent of participants from the PPOIT had SU at 8 weeks compared to $17 \%$ in the placebo group [19]. These results continue to show the effectiveness in peanut OIT; however, additional studies with these specific therapies (i.e., sealed peanut capsule and adjunctive probiotic use) would need to be replicated in order to assess the role it played in achieving the primary endpoints in comparison to other larger peanut OIT clinical trials. A larger more definitive study of PPOIT, with a probiotic-only control arm in addition to placebo, is underway.

\section{Quality of Life}

An additional area that is important to address in peanut OIT is quality of life (QoL). A number of community practices have reported QoL data in their patients treated in real-world practice, and these reports often show some benefit but should be interpreted with caution due to potential bias. Some but not all clinical trials report QoL as a secondary endpoint but importantly, the instruments used to measure QoL were not developed and validated for use in a therapeutic context. A true patient-reported outcome measure developed specifically to address clinical benefit after food immunotherapy is an urgent need. This has been pointed out by several authors, with whom we agree $[13,20,21]$.

In a prior study by Tang et al. that evaluated PPOIT vs placebo, a longitudinal impact on health-related quality of life (HRQOL) was also evaluated [22••]. Fifty-one participants completed the Food Allergy Quality of Life Questionnaire (FAQLQ-PF) and Food Allergy Independent Measure (FAIM) at pre-treatment, end-of-treatment, and 3 months after end-of-treatment; 42 of the 51 participants completed the questionnaires at 12 months post-treatment. The results showed that PPOIT was associated with a significant improvement in the FAQLQ-PF and FAIM while there was no change in the placebo group. Overall, the PPOIT group showed a benefit in the HRQOL specifically in those that acquired sustained unresponsiveness [22••]. Reier-Neilsen et al. conducted a study in 96 patients ages 5-15 years who had undergone peanut OIT and reviewed the QoL responses taken at the initial screening, during the up-dosing, and maintenance with desensitization. The children completed the validated ageadapted generic Pediatric Quality of Life Inventory questionnaire (PedsQL 4.0). Parents completed both the PedsQL 4.0 proxy-report as well as the Food Allergy Quality of LifeParental Burden (FAQL-PB) questionnaire. The results showed that the child reported QoL improvement after 2 years of OIT was not significant between the OIT group and the 
control group. However, in the child QoL reported by the parents, there was a two-fold significant change in the OIT group compared to the control group. Overall, parental proxy but not child self-reported QoL improved with OIT [23••]. In the DBRPC trial conducted by Blumchen et al., QoL was a secondary endpoint that was evaluated [8••]. Similarly, they evaluated HRQOL using the FAQLQ-PF (for mothers), FAQLQ-CF (for children), and FAQLQ-TF (for teenagers) before the initial OFC and 4 weeks after the final OFC. They also evaluated the burden of treatment (BOT) via a BOT questionnaire. The results showed a significant improvement in the HRQOL for the domains of "risk of accidental exposure" and "emotional impact" after OIT. Mothers of the peanut OIT group but not the placebo group reported an improvement in HRQOL within the domain of "social and dietary limitations." There was no significant difference in HRQOL for all domains reported by mothers in either group. In children, the peanut OIT group reported a significant improvement in HRQOL within the domains of "risk of accidental exposure" and "emotional impact" when compared with placebo. Eighty-two percent of mothers of the peanut OIT group and $100 \%$ of the placebo group reported a positive BOT. $3.7 \%$ in the OIT group would not perform OIT again. Eighty-two percent of children of the OIT group and $90 \%$ in the placebo group were positive about their treatment [8••]. Overall, there is data to suggest improvement in certain aspects of the quality of life in both the parents and children, though the effect is complex, may be more pronounced in caregivers than the children themselves, and varies over time. The beneficial effects of trial participation, such as access to experts and guided exposures through a DBPCFC (which itself has a beneficial effect on QoL independent of outcome), must be considered. More study, and development of new validated tools, is urgently needed.

\section{Predictors and Adjunctive Therapies}

\section{Baseline Predictors of Allergic Side Effects during Peanut OIT}

Peanut OIT is a promising therapy for peanut-allergic patients, but AEs may limit patient enrollment and compliance. A retrospective analysis evaluated if there were baseline predictors of allergic side effects during peanut OIT. Of 111 peanutallergic children, 104 tolerated escalation with home administration of OIT. The final study evaluated 104 participants of mostly Caucasian descent. Comorbid conditions included allergic rhinitis (AR) (46\%), asthma (44\%), and atopic dermatitis $(77 \%)$. They found that $80 \%$ of participants experienced AEs related to OIT ( $72 \%$ during build-up); $7 \%$ of events occurred in the research unit while $93 \%$ occurred while at home. Eighty-five percent of the reactions were mild, $15 \%$ moderate, and there were no severe events or SAEs. Forty-two percent experienced systemic reactions and $49 \%$ experienced GI symptoms. Participants with AR were 2.9-fold higher than those with AR to experience AEs and for every $5 \mathrm{~mm}$ increase in peanut skin prick test (SPT) there was a 1.4-fold increase in AEs. The overall presence of asthma increased AEs by 2.3fold during the maintenance phase. Overall, in a multivariate model, baseline allergic rhinitis, asthma, and peanut SPT were significant predictors of higher rates of AEs [24].

In addition to achieving the primary endpoints or peanut OIT, clinical studies are evaluating the utilization of biomarkers in explaining the heterogeneity of the clinical response to the treatment. Kukkoken et al. evaluated 60 participants ages 6-18 years in a double-blind placebo-controlled peanut challenge to assess the efficacy and safety of peanut OIT. Thirty-nine received OIT and 21 avoided peanut protein. Biomarkers including specific IgE and IgG4 were measured as well as AEs of bronchial hyper-responsiveness (BHR) to methacholine and the fractional concentrations of exhaled nitric oxide (FeNO). The results showed that $85 \%$ of the OIT participants passed the build-up phase and $67 \%$ tolerated $5 \mathrm{~g}$ of peanuts during the post-treatment challenge. There was no harmful effect on BHR to methacholine. FeNO showed no change. Regarding AEs, $77 \%$ reported AEs and $41 \%$ required antihistamines with $2.6 \%$ requiring epinephrine. During the build-up phase, five adverse events led to emergency medical contact at a rate of 4.6 per 10,000 patient days. During the follow-up period, $72 \%$ of participants consumed 100 to $2000 \mathrm{mg}$ of peanut protein on 2 to 7 days weekly and $77 \%$ of the responders continued the $800 \mathrm{mg}$ dose [25].

A recently published DBRPC clinical trial evaluated the sustained effects of long-term peanut OIT in adults and children. One hundred twenty patients ages $7-55$ years old with a confirmed peanut allergy were randomized to 3 different groups: built up to a maintenance dose of $4000 \mathrm{mg}$ through week 104 then discontinued on peanut (peanut-0 group), built up to a maintenance dose of $4000 \mathrm{mg}$ through week 104 then maintained on $300 \mathrm{mg}$ of peanut for 52 weeks (peanut-300 group), or placebo. DBPCFCs to $4000 \mathrm{mg}$ peanut protein were done at baseline and weeks 104, 117, 130, 143, and 156. The primary endpoint was the proportion of participants who reached and passed the DBPCFC to cumulative dose of $4000 \mathrm{mg}$ at weeks 104 and 117. In addition, specific biomarkers were followed including skin testing, basophil activation tests, and assays of peanut-specific $\operatorname{IgE}$ and $\mathrm{IgG}_{4}$, and total IgE. Twenty-one (35\%) of peanut-0 group participants and one (4\%) placebo group participant passed the $4000 \mathrm{mg}$ challenge at both 104 and 117 weeks (odds ratio (OR) 12.7, 95\% CI 1.8-554.8; $p=0.0024)$. There was no significant difference in success between the peanut- 0 group and then peanut-300 group at week 117 (35\% vs 54\%, $p=0.086)$, however, in all subsequent DBPCFCs, the peanut- 0 group was less likely to reach $4000 \mathrm{mg}$ than the individuals in the peanut-300 
group. A higher baseline ratio of specific $\operatorname{IgG}_{4}$ to peanutspecific IgE was associated with success only in peanut group- 0 and not peanut-300 group ( $p<0.001 ; 95 \%$ CI $0.66-$ $0.92)$ at week 117. In addition, higher peanut-specific IgE and Ara h1 and Ara h2 IgE at baseline were associated with lower odds of success at all subsequent challenges. In the peanut- 0 group, in which eight (13\%) of 60 participants passed DBPCFCs at week 156, higher baseline peanut-specific IgG4 to IgE ratio and lower Ara h 2 IgE and basophil activation responses were associated with sustained unresponsiveness. Neither the cumulative tolerated peanut dose at baseline, age, atopic comorbidity, peanut skin wheal size, were associated with higher risk of treatment failure. Overall, peanut OIT was able to desensitize patients with peanut allergy to $4000 \mathrm{mg}$ of peanut protein, however, with reduction or discontinuation, there was an increased risk of clinical reactivity [9॰].

Another recently published study evaluated how basophil sensitivity and area under the curve (AUC) to Ara h2 correlated with clinical response to OIT. Twenty-two of 30 participants (ages 7-13 years) successfully underwent treatment with OIT. Of those, nine achieved SU and 13 had transient desensitization (TD). Similar to prior studies, the pre OIT peanut-specific IgE and Ara h2-specific IgE were greater in those with TD than those with SU $(p=0.002)$, however, the ratio of Ara h2-specific IgE to total IgE was not statistically significant. A significant decrease in Ara h2-specific IgE from pre OIT occurred after OIT in those with $\operatorname{TD}(p=0.004)$ with similar trends that did not reach significance in those with SU. Additionally, Ara h2-specific IgG and $\mathrm{IgG}_{4}$ increased during OIT. The Ara h2-specific IgE levels before OIT were similar in those with TD and SU. After OIT, basophil AUC was significantly suppressed from baseline in patients with SU $(p<0.001)$ and TD $(p<0.001)$. Basophil AUC to both Ara $\mathrm{h} 2$ and peanut were similar in participants with SU and those with TD at the end of OIT; however, after 1 month of avoidance, the basophil AUC in the participants with TD rebounded as opposed to those with $\mathrm{SU}(p<0.001)[26]$.

\section{Adjunctive Therapies}

OIT is an investigative therapy that can lead to desensitization. While the hope is to allow for protection from accidental exposures and thus improve the quality of life, the process of OIT involves continued exposure to the allergen. Allergic reactions can be expected, which in some patients may cause additional anxiety and treatment dropout. Therefore, exploring alternative ways of improving patient experience while undergoing OIT is vital. In a parallel, randomized phase II controlled trial, 50 participants from ages 7-17 years consumed doses of peanut OIT at home over 24 weeks. Families attended monthly group clinic visits throughout the 7-month study. Each parent had a monthly call with the head of the patient support team, where parents could express any concerns about the treatment. The symptoms were described as "symptoms as positive signals" (SAPS) or "symptoms as side effects" (SASE). The SAPS group was encouraged to think of symptoms as a positive signal associated with increasing desensitization. By use of questionnaires, the endpoint treatment experiences evaluated were symptom anxiety, dosing experience, staff contact about symptoms, and symptoms occurrence. The endpoint treatment outcomes evaluated were adherence, time to treatment completion, biomarkers, and statistical analysis. Overall, the results found that the SAPS mindset improved treatment experience (anxiety, symptom rates) and outcomes (adherence, change in specific IgG4 levels) [27]. This suggests an important discussion that the clinician should have throughout OIT.

Another adjunctive therapy being evaluated to improve the rates of these side effects as well as improve the compliance of treatment is co-administration of a biologic. In one study, omalizumab showed that the peanut dose tolerated on initial desensitization was $250 \mathrm{mg}$ vs $22.5 \mathrm{mg}$ for the placebo group. Additionally, $79 \%$ of subjects in the omalizumab group tolerated $2000 \mathrm{mg}$ of peanut protein 6 weeks after stopping omalizumab versus $12 \%$ receiving placebo [28•]. Additionally, there are current trials evaluating the role of dupilumab in facilitated desensitization as well.

\section{Current Perspective}

Currently in practice, many allergists have adopted similar protocols based on ongoing clinical research trials in their outpatient practices. A 2019 study sought to better understand the current use of peanut allergy challenge protocols, which practices choose to offer peanut OIT, compare published and non-published approaches of peanut OIT, and the obstacles that limit peanut OIT in U.S. allergy practices [29]. This study used qualitative in-depth telephonic interviews with 34 allergists and nurse food allergy specialists across the USA. Peanut allergy was confirmed by history and skin testing with about 5-10\% utilizing diagnostic food challenges only when testing was inconclusive. They found that $94 \%$ of practices interviewed did perform peanut food challenges. Practices cited many reasons for not performing peanut OIT: perceived risk of increased potential to trigger anaphylaxis, lack of access to a validated protocol, and insurance reimbursement. The patients that were offered peanut OIT seemed to be patients ages 4-7 years; exclusion factors include patients with multiple allergies, allergic comorbidities, and/or a history of anaphylaxis. Social factors were also important including motivated and supportive families. The hope is with the recently FDA-approved product, Palforzia and accompanying protocol, allergists will feel more equipped to offer peanut OIT [29]. 


\section{Conclusions}

In summary, the major themes that have emerged from these clinical trials include a consistent demonstration that peanut OIT raises the threshold of reaction and in certain patients can achieve sustained unresponsiveness. Although many of the clinical trials were heterogeneous in their design (i.e., the patient population, the OIT product, the dosing regimen, and duration of therapy), the general suggestion is that children as young as 9 months to 17 years may benefit from this therapy. In regard to the overall safety, lower doses and slower escalation resulted in lower rates of SAEs and lower dropout rates. However, adjunctive therapy may assist in faster escalation with similar or lower rates of SAEs. Higher rates of adverse reactions may be related to specific predictors such as baseline allergic rhinitis, asthma, and peanut SPT which the provider may want to consider prior to starting therapy. Although data are sparse, the quality of life data that are available suggest improvement over time in most studies.

As discussed earlier, peanut OIT is not without risk even in those who have undergone effective desensitization. Optimizing the dosing regimens themselves and/or the selection of patients (e.g., those with low IgE levels, younger patients, milder reaction histories, adherent to dosing guidance about augmentation factors) may help to mitigate risk. Adjunctive therapy with biologics added to OIT may also prove to be helpful in further mitigating risk.

However, there are still many questions that need to be further investigated in clinical studies. These include the clinical effectiveness of peanut OIT in real-world practice including the optimal dose and duration of therapy of peanut OIT to achieve SU. Evaluation of distinct features or biomarkers in patients which may help categorize those at higher risk for reactions and guide treatment and duration of therapy. These trials were clinically significant in the pediatric setting, however, more research in the adult population would potentially allow a wider population to benefit. Testing these therapies in other key populations (i.e., ethnic and racial groups who were not widely represented in trials; those with poor asthma control and/or EoE; others) will also be helpful. Other questions include how this therapy compares in effectiveness with the other treatment modalities and adjunctive therapies. Lastly, evaluation of the real-world impact on the quality of life with the development of more specific and validated tools to better report these measurements.

In conclusion, peanut OIT appears to be a promising treatment option for peanut-allergic patients who would benefit from increasing the threshold of tolerance for protection from accidental ingestion and possibly achieving sustained unresponsiveness. With the recently FDA-approved peanut OIT product, Palforzia, the importance of ongoing research and data analysis will be crucial in assessing the effectiveness in the real-world setting.

\section{Compliance with Ethical Standards}

Conflict of Interest Dr. Vickery reports grants and personal fees from Aimmune Therapeutics, personal fees from AllerGenis, grants and personal fees from Food Allergy Research and Education, grants from Genentech, grants from DBV Technologies, grants from NIH-NIAID, grants from Regeneron, outside the submitted work. The other authors declare no conflicts of interest relevant to this manuscript.

Human and Animal Rights and Informed Consent This article does not contain any studies with human or animal subjects performed by any of the authors.

\section{References}

Papers of particular interest, published recently, have been highlighted as:

- Of importance

• Of major importance

1. Sicherer SH, Sampson HA. Food allergy: epidemiology, pathogenesis, diagnosis, and treatment. J Allergy Clin Immunol. 2014;133(2):291-307 quiz 8

2. Boyce JA, Assa'ad A, Burks AW, Jones SM, Sampson HA, Wood RA, Plaut M, Cooper SF, Fenton MJ, Arshad SH, Bahna SL, Beck LA, Byrd-Bredbenner C, Camargo CA Jr, Eichenfield L, Furuta GT, Hanifin JM, Jones C, Kraft M, Levy BD, Lieberman P, Luccioli S, McCall K, Schneider LC, Simon RA, Simons FE, Teach SJ, Yawn BP, Schwaninger JM, NIAID-Sponsored Expert Panel. Guidelines for the diagnosis and management of food allergy in the United States: summary of the NIAID-sponsored expert panel report. J Allergy Clin Immunol 2010;126(6):1105-1118.

3. Sampson HA, Aceves S, Bock SA, James J, Jones S, Lang D, Nadeau K, Nowak-Wegrzyn A, Oppenheimer J, Perry TT, Randolph C, Sicherer SH, Simon RA, Vickery BP, Wood R, Joint Task Force on Practice Parameters, Bernstein D, Blessing-Moore J, Khan D, Lang D, Nicklas R, Oppenheimer J, Portnoy J, Randolph C, Schuller D, Spector S, Tilles SA, Wallace D, Practice Parameter Workgroup, Sampson HA, Aceves S, Bock SA, James J, Jones S, Lang D, Nadeau K, Nowak-Wegrzyn A, Oppenheimer J, Perry TT, Randolph C, Sicherer SH, Simon RA, Vickery BP, Wood R Food allergy: a practice parameter update-2014. J Allergy Clin Immunol 2014; 134(5):1016-1025 e43.

4. Hofmann AM, Scurlock AM, Jones SM, Palmer KP, Lokhnygina $\mathrm{Y}$, Steele PH, et al. Safety of a peanut oral immunotherapy protocol in children with peanut allergy. J Allergy Clin Immunol. 2009;124(2):286-91 e1-6.

5. Burbank AJ, Sood P, Vickery BP, Wood RA. Oral immunotherapy for food allergy. Immunol Allergy Clin N Am. 2016;36(1):55-69.

6. Wasserman RL, Hague AR, Pence DM, Sugerman RW, Silvers SK, Rolen JG, et al. Real-world experience with peanut oral immunotherapy: lessons learned from 270 patients. J Allergy Clin Immunol Pract. 2019;7(2):418-26 e4.

7.• Chu DK, Wood RA, French S, Fiocchi A, Jordana M, Waserman S, et al. Oral immunotherapy for peanut allergy (PACE): a systematic review and meta-analysis of efficacy and safety. Lancet. 2019;393(10187):2222-32 Comment: Demonstrated moderate to high certainty that OIT treatment is very likely to result in desensitization but also increases the relative risk of allergic reactions compared to avoidance. 
8.• Investigators PGoC, Vickery BP, Vereda A, Casale TB, Beyer K, du Toit G, et al. AR101 oral immunotherapy for peanut allergy. N Eng1 J Med. 2018;379(21):1991-2001 Comment: This Phase 3 trial of AR101 was the largest DBRPC peanut OIT trial to date.

9. Blumchen K, Trendelenburg V, Ahrens F, Gruebl A, Hamelmann E, Hansen G, et al. Efficacy, safety, and quality of life in a multicenter, randomized, placebo-controlled trial of low-dose peanut oral immunotherapy in children with peanut allergy. J Allergy Clin Immunol Pract. 2019;7(2):479-91 e10. Comment: This multicenter RCT demonstrated that a low-dose regimen with very gradual up-dosing improved safety of peanut OIT, protection against real world accidents, and improved QOL in peanutallergic patients.

10. Peters RL, Allen KJ, Dharmage SC, Koplin JJ, Dang T, Tilbrook $\mathrm{KP}$, et al. Natural history of peanut allergy and predictors of resolution in the first 4 years of life: a population-based assessment. J Allergy Clin Immunol. 2015;135(5):1257-66 e1-2.

11. Vickery BP, Berglund JP, Burk CM, Fine JP, Kim EH, Kim JI, et al. Early oral immunotherapy in peanut-allergic preschool children is safe and highly effective. J Allergy Clin Immunol. 2017;139(1): $173-81$ e8.

12. Soller L, Abrams EM, Carr S, Kapur S, Rex GA, Leo S, et al. First real-world safety analysis of preschool peanut oral immunotherapy. J Allergy Clin Immunol Pract. 2019.

13. Fauquert JL, Michaud E, Pereira B, Bernard L, Gourdon-Dubois N, Rouzaire PO, et al. Peanut gastrointestinal delivery oral immunotherapy in adolescents: results of the build-up phase of a randomized, double-blind, placebo-controlled trial (PITA study). Clin Exp Allergy. 2018;48(7):862-74.

14. Tang ML, Ponsonby AL, Orsini F, Tey D, Robinson M, Su EL, et al. Administration of a probiotic with peanut oral immunotherapy: a randomized trial. J allergy Clin Immunol. 2015;135(3):73744 e8. Comment: Showed that a probiotic-containing peanut OIT formulation in late-stage clinical development was well tolerated and led to sustained clinical benefits.

15. Hsiao KC, Ponsonby AL, Axelrad C, Pitkin S, Tang MLK, Team PS. Long-term clinical and immunological effects of probiotic and peanut oral immunotherapy after treatment cessation: 4-year follow-up of a randomised, double-blind, placebo-controlled trial. Lancet Child Adolesc Health. 2017;1(2):97-105 Comment: Showed that a probiotic-containing peanut OIT formulation in late-stage clinical development was well tolerated and led to sustained clinical benefits.

16. Duca B, Patel N, Turner PJ. GRADE-ing the benefit/risk equation in food immunotherapy. Curr Allergy Asthma Rep. 2019;19(6):30 Comment: A systematic review that comprehensively discusses the current knowledge gaps in the field and proposes a framework to understand the benefit/risk of the treatment.

17. Chong KW, Turner PJ. Food allergy desensitisation: a hard nut to crack? Arch dis child. 2019.

18. Dunn Galvin A, McMahon S, Ponsonby AL, Hsiao KC, Tang MLK, Team Ps. The longitudinal impact of probiotic and peanut oral immunotherapy on health-related quality of life. Allergy. 2018;73(3):560-568.

19. Reier-Nilsen T, Carlsen KCL, Michelsen MM, Drottning S, Carlsen $\mathrm{KH}$, Zhang $\mathrm{C}$, et al. Parent and child perception of quality of life in a randomized controlled peanut oral immunotherapy trial. Pediatr Allergy Immunol. 2019.

20. Virkud YV, Burks AW, Steele PH, Edwards LJ, Berglund JP, Jones SM, et al. Novel baseline predictors of adverse events during oral immunotherapy in children with peanut allergy. J Allergy Clin Immunol. 2017;139(3):882-8 e5.
21. Kukkonen AK, Uotila R, Malmberg LP, Pelkonen AS, Makela MJ. Double-blind placebo-controlled challenge showed that peanut oral immunotherapy was effective for severe allergy without negative effects on airway inflammation. Acta Paediatr. 2017;106(2):274 81.

22.• Chinthrajah RS, Purington N, Andorf S, Long A, O'Laughlin KL, Lyu SC, et al. Sustained outcomes in oral immunotherapy for peanut allergy (POISED study): a large, randomised, double-blind, placebo-controlled, phase 2 study. Lancet. 2019; Comment: The largest trial to prospectively address the rate of clinical remission after treatment with peanut OIT using three different regimens.

23.• Patil SU, Steinbrecher J, Calatroni A, Smith N, Ma A, Ruiter B, et al. Early decrease in basophil sensitivity to Ara h 2 precedes sustained unresponsiveness after peanut oral immunotherapy. J Allergy Clin Immunol. 2019; Comment: Demonstrated that basophil activation testing three months into treatment, and at the time of treatment cessation, predicts clinical remission, suggesting its use as a biomarker.

24. Howe LC, Leibowitz KA, Perry MA, Bitler JM, Block W, Kaptchuk TJ, et al. Changing patient mindsets about non-lifethreatening symptoms during oral immunotherapy: a randomized clinical trial. J Allergy Clin Immunol Pract. 2019;7(5):1550-9.

25. MacGinnitie AJ, Rachid R, Gragg H, Little SV, Lakin P, Cianferoni A, et al. Omalizumab facilitates rapid oral desensitization for peanut allergy. J Allergy Clin Immunol. 2017;139(3):873-81 e8.

26. Blaiss MS, Tilles S, Petroni D, Zigmont E, Cassese M, Kwak B, et al. Current management and use of oral immunotherapy in the United States for patients with peanut allergy. Allergy Asthma Proc. 2019.

27. Wasserman RL, Jones DH, Windom HH. Oral immunotherapy for food allergy: the FAST perspective. Ann Allergy Asthma Immunol. 2018;121(3):272-5.

28. Wasserman RL, Jones DH, Windom HH, Meeting F. Reaching for best practices in food oral immunotherapy: report on the second annual Food Allergy Support Team meeting. Ann Allergy Asthma Immunol. 2019;123(2):129-30 e3. Comment: The leading clinical practitioners of OIT (not only for peanut) review their practices, giving some insights into the real-world experience of treating patients.

29. Bird JA, Feldman M, Arneson A, Dougherty I, Brown LS, Burk $\mathrm{CM}$, et al. Modified peanut oral immunotherapy protocol safely and effectively induces desensitization. J Allergy Clin Immunol Pract. 2015;3(3):433-5 e1-3.

30. Narisety SD, Frischmeyer-Guerrerio PA, Keet CA, Gorelik M, Schroeder J, Hamilton RG, et al. A randomized, double-blind, placebo-controlled pilot study of sublingual versus oral immunotherapy for the treatment of peanut allergy. J Allergy Clin Immunol. 2015;135(5):1275-82 e1-6.

31. Vickery BP, Scurlock AM, Kulis M, Steele PH, Kamilaris J, Berglund JP, et al. Sustained unresponsiveness to peanut in subjects who have completed peanut oral immunotherapy. J Allergy Clin Immunol. 2014;133(2):468-75.

32. Anagnostou K, Islam S, King Y, Foley L, Pasea L, Bond S, et al. Assessing the efficacy of oral immunotherapy for the desensitisation of peanut allergy in children (STOP II): a phase 2 randomised controlled trial. Lancet. 2014;383(9925):1297-304.

Publisher's Note Springer Nature remains neutral with regard to jurisdictional claims in published maps and institutional affiliations. 